\title{
The incidence of dorsal and pelvis asymmetries in school-age children with hip dysplasia in the neonatal-infancy period Marek Kluszczyński
}

Address: Rehabilitation Ward, Provincial Specialist Hospital in Czestochowa, Poland

Email: Marek Kluszczyński - marek.kluszczynski@mechatronika.edu.pl

from 4th International Conference on Conservative Management of Spinal Deformities

Boston, MA, USA. 13-16 May 2007

Published: 12 October 2007

Scoliosis 2007, 2(SuppI I):S33 doi:10.II86/1748-7|6I-2-SI-S33

This abstract is available from: http://www.scoliosisjournal.com/content/2/SI/S33

(c) 2007 Kluszczyński; licensee BioMed Central Ltd.

\section{Objective}

The aim was the comparative analysis of the incidence of dorsal and pelvis asymmetries in school-age children with hip dysplasia in the infancy period.

\section{Study design}

Fifty children aged from four to fourteen years, diagnosed with hip dysplasia in the infancy period, were included in the study. Using a Rippstein plurimeter [1], dorsal and pelvis asymmetries were examined. The control group included fifty randomly chosen children aged from twelve to sixteen years.

\section{Results}

Dorsal asymmetry was found in ninety percent of test group children, including a small asymmetry of 2-4 Bunnell degrees [2] in seventy-eight percent. In the control group, dorsal asymmetry was found in fifty percent of children, including a small asymmetry in forty-six percent. Pelvis asymmetry was found in eighty percent of test group children, predominantly an eight-type deformation with a right-side anterior superior iliac spine (ASIS) lowering in sixty-six percent and a left-side posterior superior iliac spine (PSIS) lowering in forty-six percent of children. In the control group, pelvis asymmetry was found only in forty-two percent of children.

\section{Conclusion}

A statistically significant, higher incidence of dorsal and pelvis asymmetries is found in children with single-hip dysplasia in their infancy period as against the children who did not experience dysplasia.

\section{References}

I. Green S, Buchbinder R, Forbes A, Bellamy N: A standardized protocol for measurement of range of movement of the shoulder using the Plurimeter-V inclinometer and assessment of its intrarater and interrater reliability. Arthritis Care Res 1998, I I:43-52.

2. Bunnell WP: An objective criterion for scoliosis screening. J Bone Joint Surg 1984, 66A:I38I-1387. 